\title{
AN ANALYSIS FOR THERMAL-BENDING STRESSES IN AN ANNULAR SECTOR PLATE BY THE THEORY OF MODERATELY THICK PLATES
}

\author{
By Isamu A. OKUMURA*, Yuya HONDA** and Jin YOSHIMURA***
}

\begin{abstract}
An analysis for thermal-bending stresses in an annular sector plate with the moderate thickness is carried out by the theory of moderately thick plates, together with the thermoelastic displacement potential. The temperature field in the plate is considered a steady-state prescribed to surface temperatures on the top and bottom faces and on all the edges, of the plate. Mechanical boundary conditions on the top and bottom faces and on the edges are considered stress-free and simple support. A three-dimensional elasticity solution as a particular solution and a plane and generalized plane stress solutions as a homogeneous solution are used. Numerical results for thermal-bending stresses and displacements in the plate are presented. The influence of the thickness-width ratio on the stresses is examined.
\end{abstract}

Keywords: thermoelasticity, thermal stress, bending, annular sector plate

\section{INTRODUCTION}

A number of studies on bending of plates with the comparatively large thicknesses called thick plates have been done by many researchers on the basis of the theory of elasticity or what is called the theory of moderately thick plates. The study based upon the theory of moderately thick plates appears to have been done first by Woinowsky-Krieger ${ }^{1)}$. After that, as to representative researchers, Love ${ }^{2)}$, Reissner ${ }^{3)}$, Mindlin $^{4)}$ and Lo et al. ${ }^{5)}$ have proposed the analytical theories which are simple and highly precise. Though the studies on bending of thick plates have been offensive in the long run, they have been few lately and seems to have passed the peak concerning isotropic, thick plates.

Though the bending problems of thick plates have been mainly dealt with prescribed surface loads, bending of thick plates with the temperature change in the plates is also important. Bending of thin plates by heat seems to have been already systematized and are stated in Nowinski's book ${ }^{6}$ too. Furthermore, the studies on analyses for thermal stresses in two-dimensional or three-dimensional discs are somewhat found. Tauchert and Aköz ${ }^{7)}$, and Ishikawa et al. ${ }^{8)}$ have analyzed steady-state thermal stresses in an orthotropic, infinite band slab in a state of plane strain. Noda et al. ${ }^{9)}$ have analyzed axi-symmetric, transient thermal stresses in a circular thick disc, and Sugano and Kimoto ${ }^{10)}$ have analyzed axially asymmetric, transient thermal stresses in an annular disc with the variable thickness. Furthermore, a few studies on thermal stress analyses for transversely isotropic, thick discs have been lately found with the latest development of anisotropic materials. Noda and Takeuti ${ }^{11}$ have analyzed axi-symmetric, transient thermal stresses in a transversely isotropic circular thick disc, and Sugano ${ }^{12 l}$ has analyzed axi-symmetric,

* Member of JSCE, Dr. Eng. , Professor, Department of Civil Engineering, Kitami Institute of Technology (165, Koen-cho, Kitami).

** Member of JSCE, Professor, Dean of Engineering, Department of Civil Engineering, Hokkai-Gakuen University (26-11, Chuo-ku, Sapporo).

*** Member of JSCE, Dr. Eng., Professor, Department of Civil Engineering, University of Hokkaido (13-8, Kita-ku, Sapporo) . 
transient thermal stresses in a transversely isotropic infinite thick disc with a cylindrical hole. However, the studies on bending of thick plates by heat, without distinction of coordinate systems, are hardly found at the present time. Though a three-dimensional analysis for thermal stresses even in thick plates is the theoretically most exact method of analysis, numerical calculations become increasingly difficult as the thicknesses of plates decrease. When attention is paid to this point, the simple theory of thick plates to analyze bending of plates with the moderate thicknesses by heat is very important. Furthermore, the data on thermal-bending stresses in annular sector thick plates as seen in the slabs of curved bridges are very few at the present time and so are highly significant for engineering.

From the above point of view, this paper is concerned with an analysis for thermal-bending stresses in an annular sector plate with the moderate thickness by the theory of moderately thick plates proposed by the authors in a previous paper ${ }^{13)}$, together with the thermoelastic displacement potential. The temperature field in the annular sector plate is considered a steady-state prescribed to surface temperatures on the top and bottom faces and on all the edges, of the plate. Mechanical boundary conditions on the top and bottom faces and on the edges are considered stress-free and simple support, respectively. A three-dimensional elasticity solution as a particular solution and a plane and generalized plane stress solutions as a homogeneous solution are used, because the boundary conditions on the top and bottom faces and on the edges can not be satisfied by the thermoelastic displacement potential alone. The method of analysis presented in this paper is applicable to bending of circular and annular thick plates by heat and is considerably extensive in its application. Furthermore, the method is applicable to bending of rectangular thick plates by heat, if a particular and homogeneous solutions in Cartesian coordinates are used.

\section{STEADY-STATE TEMPERATURE FIELD}

When cylindrical coordinates $(r, \theta, z)$ are used, the governing equation for steady-state heat conduction in isotropic solids is as follows:

$\nabla^{2} T=0$,

in which $T$ is the temperature change, and

$$
\nabla^{2} \equiv \frac{\partial^{2}}{\partial r^{2}}+\frac{1}{r} \frac{\partial}{\partial r}+\frac{1}{r^{2}} \frac{\partial^{2}}{\partial \theta^{2}}+\frac{\partial^{2}}{\partial z^{2}} \text {. }
$$

We take the cylindrical coordinates of an annular sector plate as Fig. 1 and consider the temperature $T$ to be even in $\theta$. Then, from Eq. (1), the steady-state temperature $T$ is expressed as

$$
\begin{aligned}
& T=\sum_{t=1}^{\infty} \sum_{s=1}^{\infty} \cos m \theta\left[J_{m}\left(\alpha_{m s} r\right)\left(N_{t s}^{(1)} \cosh \alpha_{m s} z+R_{t s}^{(1)} \sinh \alpha_{m s} z\right)+Y_{m}\left(\alpha_{m s} r\right)\left(N_{t s}^{(2)} \cosh \alpha_{m s} z\right.\right. \\
& \left.\left.+R_{t s}^{(2)} \sinh \alpha_{m s} z\right)\right]+\sum_{t=1}^{\infty} \sum_{n=1}^{\infty} \cos m \theta \cos \bar{\beta}_{n} z\left[S_{t n}^{(1)} I_{m}\left(\bar{\beta}_{n} r\right)+S_{t n}^{(2)} K_{m}\left(\bar{\beta}_{n} r\right)\right],
\end{aligned}
$$

in which $N_{t s}^{(1)}, \cdots, S_{t n}^{(2)}$ are arbitrary constants to be determined from boundary conditions. Furthermore, $J_{m}\left(\alpha_{m s} r\right)$ and $Y_{m}\left(\alpha_{m s} r\right)$ denote Bessel functions of the first and second kinds, of order $m$, respectively, $I_{m}\left(\bar{\beta}_{n} r\right)$ and $K_{m}\left(\bar{\beta}_{n} r\right)$ denote the modified Bessel functions of the first and second kinds, of order $m$, respectively, and

$$
\begin{gathered}
m=\frac{t \pi}{\gamma} \quad(t=1,3, \cdots) ; \\
\alpha_{m s}=\frac{\lambda_{m s}}{a} \quad(s=1,2, \cdots) ;
\end{gathered}
$$

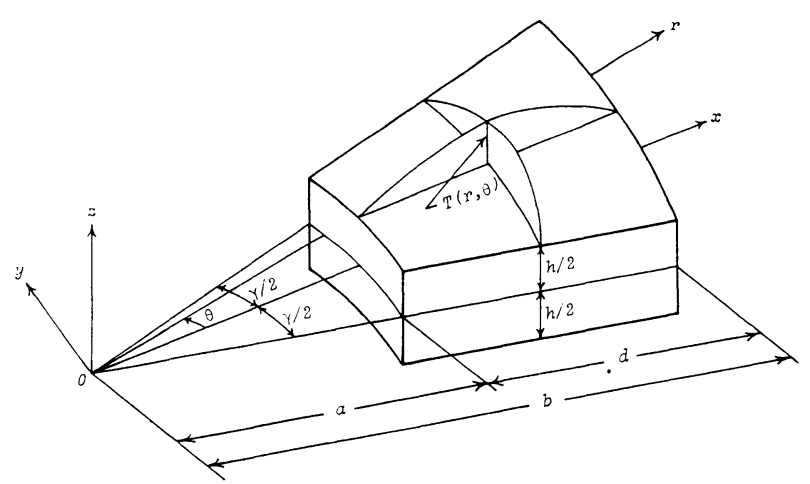

Fig. 1 Coordinate system of annular sector plate. 
$\bar{\beta}_{n}=\frac{n \pi}{h} \quad(n=1,2, \cdots)$.

We consider the following boundary conditions for the temperature field in the annular sector plate :

on $z=h / 2, \quad T=g(r, \theta)$,

on $z=-h / 2, \quad T=0$,

on $r=a$ and $r=b, \quad T=0$,

on $\theta= \pm \gamma / 2, \quad T=0$,

in which $g(r, \theta)$ denote the prescribed surface temperature on the top face. The boundary condition of Eq. (4·d) is self-evidently satisfied in Eq. (2) with the aid of Eq. (3・a). Here, we put the arbitrary constants in Eq. (2) in the form

$$
N_{t s}^{(2)}=-\varepsilon_{m s} N_{t s}^{(1)}, \quad R_{t s}^{(2)}=-\varepsilon_{m s} R_{t s}^{(1)}, \quad S_{t n}^{(1)}=S_{t n}^{(2)}=0,
$$

in which

$$
\varepsilon_{m s}=\frac{J_{m}\left(\alpha_{m s} a\right)}{Y_{m}\left(\alpha_{m s} a\right)}=\frac{J_{m}\left(\lambda_{m s}\right)}{Y_{m}\left(\lambda_{m s}\right)} \text {. }
$$

By making use of Eqs. $(5 \cdot a \sim c), T$ is expressed as

$$
T=\sum_{t=1}^{\infty} \sum_{s=1}^{\infty} \cos m \theta C_{m}\left(\alpha_{m s} r\right)\left(N_{t s}^{(1)} \cosh \alpha_{m s} z+R_{t s}^{(1)} \sinh \alpha_{m s} z\right),
$$

in which $C_{m}\left(\alpha_{m s} r\right)$ denotes a cylinder function of order $m$ as defined in the form

$$
C_{m}\left(\alpha_{m s} r\right)=J_{m}\left(\alpha_{m s} r\right)-\varepsilon_{m s} Y_{m}\left(\alpha_{m s} r\right) \text {. }
$$

The boundary condition of Eq. $(4 \cdot \mathrm{c})$ on $r=a$ is already satisfied by Eq. (8). From the boundary condition on $r=b$, we have the following transcendental equation from which the root $\lambda_{m s}$ is determined :

$$
J_{m}\left(x \lambda_{m s}\right) Y_{m}\left(\lambda_{m s}\right)-J_{m}\left(\lambda_{m s}\right) Y_{m}\left(x \lambda_{m s}\right)=0, \quad x=b / a \text {. }
$$

In order to satisfy the boundary condition of $\mathrm{Eq}$. $(4 \cdot \mathrm{a})$, we expand the prescribed surface temperature $g(r, \theta)$ into the following Fourier-Bessel series:

$$
g(r, \theta)=\sum_{t=1}^{\infty} \sum_{s=1}^{\infty} f_{t s} \cos m \theta C_{m}\left(\alpha_{m s} r\right),
$$

in which

$$
f_{t s}=\frac{\int_{-\gamma / 2}^{\gamma / 2} \int_{a}^{b} g(r, \theta) r C_{m}\left(\alpha_{m s} r\right) \cos m \theta d r d \theta}{\int_{-\gamma / 2}^{\gamma / 2} \cos ^{2} m \theta d \theta \cdot \int_{a}^{b} r C_{m}^{2}\left(\alpha_{m s} r\right) d r} .
$$

From Eq. (10) and the boundary conditions of Eqs. $(4 \cdot a, b)$, the arbitrary constants are determined in the form

$$
N_{t s}^{(1)}=\frac{f_{t s}}{2 \cosh \zeta_{m s}}, \quad R_{t s}^{(1)}=\frac{f_{t s}}{2 \sinh \zeta_{m s}}, \quad \zeta_{m s}=\frac{\alpha_{m s} h}{2} .
$$

By substituting the values of Eqs. $(12 \cdot a \sim c)$ into Eq. ( 7 ), the temperature $T$ is exactly determined in the form

$$
T=\sum_{t=1}^{\infty} \sum_{s=1}^{\infty} \frac{f_{t s}}{2} \cos m \theta C_{m}\left(\alpha_{m s} r\right)\left(\frac{\cosh \alpha_{m s} z}{\cosh \zeta_{m s}}+\frac{\sinh \alpha_{m s} z}{\sinh \zeta_{m s}}\right)
$$

\section{DISPLACEMENT AND STRESS FIELD}

A particular and homogeneous solutions are needed to satisfy mechanical boundary conditions of stress-free on the top and bottom faces and of simple support on all the edges.

\section{(1) Particular solution}

As a particular solution, we shall use a three-dimensional elasticity solution ${ }^{14)}$ proposed by one of the authors. Let $u_{r}, u_{\theta}$ and $u_{z}$ be components of displacement, the solution is expressed as

$$
2 G u_{r}=\frac{\partial}{\partial r}\left[\phi_{0}+r \frac{\partial \phi_{1}}{\partial r}+z \frac{\partial \phi_{3}}{\partial z}-4(1-\nu) \phi_{1}\right]+\frac{2}{r} \frac{\partial \vartheta_{z}}{\partial \theta}+\frac{\partial \chi}{\partial r},
$$




$$
\begin{aligned}
& 2 G u_{\theta}=\frac{1}{r} \frac{\partial}{\partial \theta}\left[\phi_{0}+r \frac{\partial \phi_{1}}{\partial r}+z \frac{\partial \phi_{3}}{\partial z}-4(1-\nu) \phi_{1}\right]-2 \frac{\partial \vartheta_{z}}{\partial r}+\frac{1}{r} \frac{\partial \chi}{\partial \theta}, \\
& 2 G u_{z}=\frac{\partial}{\partial z}\left[\phi_{0}+r \frac{\partial \phi_{1}}{\partial r}+z \frac{\partial \phi_{3}}{\partial z}-4(1-\nu) \phi_{3}\right]+\frac{\partial \chi}{\partial z},
\end{aligned}
$$

in which $G$ and $\nu$ denote the shear modulus and Poisson's ratio, respectively, and

$$
\nabla^{2} \phi_{0}=0, \quad \nabla^{2} \phi_{1}=0, \quad \nabla^{2} \phi_{3}=0, \quad \nabla^{2} \vartheta_{z}=0, \quad \nabla^{2} \chi=\frac{\alpha E}{1-\nu} T .
$$

The function $\chi$ in Eq. $(15 \cdot \mathrm{e})$ is called the thermoelastic displacement potential, and $\alpha$ and $E$ denote the coefficient of linear thermal expansion and Young's modulus, respectively. If the potential function $\phi_{1}$ is excluded on account of inutility for the particular solution, from Eqs. $(15 \cdot a, c, d)$ the potential functions are expressed as

$$
\begin{aligned}
& \phi_{0}=\sum_{t=1}^{\infty} \sum_{s=1}^{\infty} \cos m \theta\left[J_{m}\left(\alpha_{m s} r\right)\left(A_{t s}^{(1)} \cosh \alpha_{m s} z+L_{t s}^{(1)} \sinh \alpha_{m s} z\right)+Y_{m}\left(\alpha_{m s} r\right)\left(A_{t s}^{(2)} \cosh \alpha_{m s} z\right.\right. \\
& \left.+L_{t s}^{(2)} \sinh \alpha_{m s} z\right) \text {, } \\
& \phi_{3}=\sum_{t=1}^{\infty} \sum_{s=1}^{\infty} \cos m \theta\left[J_{m}\left(\alpha_{m s} r\right)\left(C_{t s}^{(1)} \cosh \alpha_{m s} z+M_{t s}^{(1)} \sinh \alpha_{m s} z\right)+Y_{m}\left(\alpha_{m s} r\right)\left(C_{t s}^{(2)} \cosh \alpha_{m s} z\right.\right. \\
& \left.+M_{t s}^{(2)} \sinh \alpha_{m s} z\right) \text {, } \\
& \vartheta_{z}=\sum_{t=1}^{\infty} \sum_{s=1}^{\infty} \sin m \theta\left[J_{m}\left(\alpha_{m s} r\right)\left(B_{t s}^{(1)} \cosh \alpha_{m s} z+G_{t s}^{(1)} \sinh \alpha_{m s} z\right)+Y_{m}\left(\alpha_{m s} r\right)\left(B_{t s}^{(2)} \cosh \alpha_{m s} z\right.\right. \\
& \left.\left.+G_{t s}^{(2)} \sinh \alpha_{m s} z\right)\right]+\sin \beta z \sum_{t=1}^{\infty} \sin m \theta\left[E_{t}^{(1)} I_{m}(\beta r)+E_{t}^{(2)} K_{m}(\beta r)\right],
\end{aligned}
$$

in which $A_{t s}^{(1)}, \cdots, E_{t}^{(2)}$ are arbitrary constants to be determined from boundary conditions on the top and bottom faces and on the edges, and $\beta=\pi / h$. If the complementary function of $\chi$ in Eq. $(15 \cdot e)$ is excluded on account of dependence on $\phi_{0}$, by substituting $T$ of Eq. (13) into the right-hand side of Eq. $(15 \cdot e)$, the particular integral of $\chi$ is expressed as

$$
\chi=\frac{\alpha E}{4(1-\nu)} \sum_{t=1}^{\infty} \sum_{s=1}^{\infty} \frac{f_{t s}}{\alpha_{m s}^{2}} \cos m \theta C_{m}\left(\alpha_{m s} r\right)\left(\alpha_{m s} z\right)\left(\frac{\sinh \alpha_{m s} z}{\cosh \zeta_{m s}}+\frac{\cosh \alpha_{m s} z}{\sinh \zeta_{m s}}\right) .
$$

In order to express components of displacement and stress in the cylinder function defined in Eq. ( 8 ), we put the arbitrary constants in Eqs. $(16 \cdot a \sim c)$ in the form

$$
\begin{aligned}
& A_{t s}^{(2)}=-\varepsilon_{m s} A_{t s}^{(1)}, \quad L_{t s}^{(2)}=-\varepsilon_{m s} L_{t s}^{(1)}, \quad C_{t s}^{(2)}=-\varepsilon_{m s} C_{t s}^{(1)}, \quad M_{t s}^{(2)}=-\varepsilon_{m s} M_{t s}^{(1)}, \quad B_{t s}^{(2)}=-\varepsilon_{m s} B_{t s}^{(1)} ; \\
& G_{t s}^{(2)}=-\varepsilon_{m s} G_{t s}^{(1)}
\end{aligned}
$$

Hooke's law taking account of heat is as follows:

$$
\begin{aligned}
& \sigma_{r r}=2 G\left(\varepsilon_{r r}+\frac{\nu}{1-2 \nu} e\right)-\frac{\alpha E T}{1-2 \nu}, \quad \sigma_{\theta \theta}=2 G\left(\varepsilon_{\theta \theta}+\frac{\nu}{1-2 \nu} e\right)-\frac{\alpha E T}{1-2 \nu} ; \\
& \sigma_{z z}=2 G\left(\varepsilon_{z z}+\frac{\nu}{1-2 \nu} e\right)-\frac{\alpha E T}{1-2 \nu}, \quad \sigma_{\theta z}=2 G \varepsilon_{\theta z}, \quad \sigma_{z r}=2 G \varepsilon_{z r}, \quad \sigma_{r \theta}=2 G \varepsilon_{r \theta},
\end{aligned}
$$

in which $\sigma_{r r}, \cdots, \sigma_{r \theta}$ are components of stress, $\varepsilon_{r r}, \cdots, \varepsilon_{r \theta}$ are components of strain, and $e$ is the cubical dilatation. By making use of Eqs. $(18 \cdot a \sim f)$ and substituting Eqs. $(16 \cdot a \sim c)$ and (17) into Eqs. $(14 \cdot a \sim$ c), we obtain expressions for the components of displacement. If we obtain expressions for the components of strain from the expressions for the components of displacement, we obtain expressions for the components of stress from Eqs. $(19 \cdot a \sim f)$. As examples of the expressions for the components of stress, the expressions for $\sigma_{z z}, \sigma_{\theta z}$ and $\sigma_{z r}$ with the superscript $(p)$ are

$$
\begin{aligned}
\sigma_{z z}^{(p)}= & \sum_{t=1}^{\infty} \sum_{s=1}^{\infty} \alpha_{m s}^{2} \cos m \theta C_{m}\left(\alpha_{m s} r\right)\left\{\left[A_{t s}^{(1)}-2(1-\nu) C_{t s}^{(1)}\right] \cosh \alpha_{m s} z\right. \\
& \left.+\left[L_{t s}^{(1)}-2(1-\nu) M_{t s}^{(1)}\right] \sinh \alpha_{m s} z+\alpha_{m s} z\left(C_{t s}^{(1)} \sinh \alpha_{m s} z+M_{t s}^{(1)} \cosh \alpha_{m s} z\right)\right\} \\
& +\frac{\alpha E}{4(1-\nu)} \sum_{t=1}^{\infty} \sum_{s=1}^{\infty} f_{t s} \cos m \theta C_{m}\left(\alpha_{m s} r\right)\left(\alpha_{m s} z\right)\left(\frac{\sinh \alpha_{m s} z}{\cosh \zeta_{m s}}+\frac{\cosh \alpha_{m s} z}{\sinh \zeta_{m s}}\right),
\end{aligned}
$$




$$
\begin{aligned}
& \sigma_{\theta z}^{(p)}=\sum_{t=1}^{\infty} \sum_{s=1}^{\infty}\left(-\frac{\alpha_{m s}^{2}}{2}\right) \sin m \theta\left\{C _ { m - 1 } ( \alpha _ { m s } r ) \left[\left\{A_{t s}^{(1)}+B_{t s}^{(1)}-(1-2 \nu) C_{t s}^{(1)}\right\} \sinh \alpha_{m s} z\right.\right. \\
& \left.+\left\{L_{t s}^{(1)}+G_{t s}^{(1)}-(1-2 \nu) M_{t s}^{(1)}\right\} \cosh \alpha_{m s} z+\alpha_{m s} z\left(C_{t s}^{(1)} \cosh \alpha_{m s} z+M_{t s}^{(1)} \sinh \alpha_{m s} z\right)\right] \\
& +C_{m+1}\left(\alpha_{m s} r\right)\left[\left\{A_{t s}^{(1)}-B_{t s}^{(1)}-(1-2 \nu) C_{t s}^{(1)}\right\} \sinh \alpha_{m s} z\right. \\
& \left.\left.+\left\{L_{t s}^{(1)}-G_{t s}^{(1)}-(1-2 \nu) M_{t s}^{(1)}\right\} \cosh \alpha_{m s} z+\alpha_{m s} z\left(C_{t s}^{(1)} \cosh \alpha_{m s} z+M_{t s}^{(1)} \sinh \alpha_{m s} z\right)\right]\right\} \\
& -\frac{\beta^{2}}{2} \cos \beta z \sum_{t=1}^{\infty} \sin m \theta\left\{E_{t}^{(1)}\left[I_{m-1}(\beta r)+I_{m+1}(\beta r)\right]-E_{t}^{(2)}\left[K_{m-1}(\beta r)+K_{m+1}(\beta r)\right]\right\} \\
& +\frac{\alpha E}{4(1-\nu)} \sum_{t=1}^{\infty} \sum_{s=1}^{\infty}\left(-m f_{t s}\right) \sin m \theta \frac{C_{m}\left(\alpha_{m s} r\right)}{\alpha_{m s} r}\left[\frac{1}{\cosh \zeta_{m s}}\left(\sinh \alpha_{m s} z+\alpha_{m s} z \cosh \alpha_{m s} z\right)\right. \\
& \left.+\frac{1}{\sinh \zeta_{m s}}\left(\cosh \alpha_{m s} z+\alpha_{m s} z \sinh \alpha_{m s} z\right)\right] \text {, } \\
& \sigma_{z r}^{(p)}=\sum_{t=1}^{\infty} \sum_{s=1}^{\infty} \frac{\alpha_{m s}^{2}}{2} \cos m \theta\left\{C _ { m - 1 } ( \alpha _ { m s } r ) \left[\left\{A_{t s}^{(1)}+B_{t s}^{(1)}-(1-2 \nu) C_{t s}^{(1)}\right\} \sinh \alpha_{m s} z\right.\right. \\
& \left.+\left\{L_{t s}^{(1)}+G_{t s}^{(1)}-(1-2 \nu) M_{t s}^{(1)}\right\} \cosh \alpha_{m s} z+\alpha_{m s} z\left(C_{t s}^{(1)} \cosh \alpha_{m s} z+M_{t s}^{(1)} \sinh \alpha_{m s} z\right)\right] \\
& -C_{m+1}\left(\alpha_{m s} r\right)\left[\left\{A_{t s}^{(1)}-B_{t s}^{(1)}-(1-2 \nu) C_{t s}^{(1)}\right\} \sinh \alpha_{m s} z+\left\{L_{t s}^{(1)}-G_{t s}^{(1)}-(1-2 \nu) M_{t s}^{(1)}\right\} \cosh \alpha_{m s} z\right. \\
& \left.\left.+\alpha_{m s} z\left(C_{t s}^{(1)} \cosh \alpha_{m s} z+M_{t s}^{(1)} \sinh \alpha_{m s} z\right)\right]\right\}+\frac{\beta^{2}}{2} \cos \beta z \sum_{t=1}^{\infty} \cos m \theta\left\{E_{t}^{(1)}\left[I_{m-1}(\beta r)-I_{m+1}(\beta r)\right]\right. \\
& \left.-E_{t}^{(2)}\left[K_{m-1}(\beta r)-K_{m+1}(\beta r)\right]\right\}+\frac{\alpha E}{4(1-\nu)} \sum_{t=1}^{\infty} \sum_{s=1}^{\infty} f_{t s} \cos m \theta\left[\frac{m}{\alpha_{m s} r} C_{m}\left(\alpha_{m s} r\right)\right. \\
& \left.-C_{m+1}\left(\alpha_{m s} r\right)\right]\left[\frac{1}{\cosh \zeta_{m s}}\left(\sinh \alpha_{m s} z+\alpha_{m s} z \cosh \alpha_{m s} z\right)\right. \\
& \left.+\frac{1}{\sinh \zeta_{m s}}\left(\cosh \alpha_{m s} z+\alpha_{m s} z \sinh \alpha_{m s} z\right)\right] \text {. }
\end{aligned}
$$

The expressions with the single summation notation as seen in Eqs. $(20 \cdot \mathrm{b}, \mathrm{c})$ are anti-symmetric solutions with respect to the middle plane, i. e., $z=0$.

\section{(2) Homogeneous solution}

We shall use a plane and generalized plane stress solutions to thick plates in the cylindrical coordinates as a homogeneous solution. The form of these solutions has been previously reported by one of the authors ${ }^{15)}$. The concrete expressions for the components of displacement and stress shall be given here. For economy of space, the expressions for the components of displacement and four components of stress, i. e., $\sigma_{r r}, \sigma_{z z}, \sigma_{\theta z}$ and $\sigma_{z r}$ are presented.

a) Plane stress solution: The expressions for those components with the superscript $(h, 1)$ are

$$
\begin{aligned}
& 2 G u_{r}^{(h, 1)}=-\sum_{t=1}^{\infty} \cos m \theta\left\{m\left(P_{t}^{(1)} r^{m-1}-P_{t}^{(2)} r^{-m-1}\right)-P_{t}^{(3)} r^{m-1}\left[r^{2}\left\{\frac{4}{1+\nu}-(m+2)\right\}\right.\right. \\
& \left.\left.+\frac{2 \nu}{1+\nu} m(m+1) z^{2}\right]-P_{t}^{(4)} r^{-m-1}\left[r^{2}\left\{\frac{4}{1+\nu}+(m-2)\right\}+\frac{2 \nu}{1+\nu} m(m-1) z^{2}\right]\right\}, \\
& 2 G u_{\theta}^{(h, 1)}=\sum_{t=1}^{\infty} \sin m \theta\left\{m\left(P_{t}^{(1)} r^{m-1}+P_{t}^{(2)} r^{-m-1}\right)+P_{t}^{(3)} r^{m-1}\left[r^{2}\left(\frac{4}{1+\nu}+m\right)-\frac{2 \nu}{1+\nu} m(m+1) z^{2}\right]\right. \\
& \left.-P_{t}^{(4)} r^{-m-1}\left[r^{2}\left(\frac{4}{1+\nu}-m\right)-\frac{2 \nu}{1+\nu} m(m-1) z^{2}\right]\right\}, \\
& 2 G u_{z}^{(h, 1)}=-\frac{4 \nu}{1+\nu} z \sum_{t=1}^{\infty} \cos m \theta\left[(m+1) P_{t}^{(3)} r^{m}-(m-1) P_{t}^{(4)} r^{-m}\right] \text {, } \\
& \sigma_{r r}^{(h, 1)}=-\sum_{t=1}^{\infty} \cos m \theta\left\{m(m-1) P_{t}^{(1)} r^{m-2}+m(m+1) P_{t}^{(2)} r^{-m-2}+(m+1) P_{t}^{(3)} r^{m-2}\left[r^{2}(m-2)\right.\right. \\
& \left.\left.-\frac{2 \nu}{1+\nu} m(m-1) z^{2}\right]+(m-1) P_{t}^{(4)} r^{-m-2}\left[r^{2}(m+2)+\frac{2 \nu}{1+\nu} m(m+1) z^{2}\right]\right\}, \\
& \sigma_{z z}^{(h, 1)}=\sigma_{\theta z}^{(h, 1)}=\sigma_{z r}^{(h, 1)}=0,
\end{aligned}
$$

in which $P_{t}^{(1)}, \cdots, P_{t}^{(4)}$ are arbitrary constants to be determined from the boundary conditions on the edges. 
b) Generalized plane stress solution: The expressions for those components with the superscript $(h, 2)$ are

$$
\begin{aligned}
& 2 G u_{r}^{(h, 2)}=-z \sum_{t=1}^{\infty} \cos m \theta\left\{m\left(Q_{t}^{(1)} r^{m-1}-Q_{t}^{(2)} r^{-m-1}\right)+Q_{t}^{(3)} r^{m-1}\left[(m+2) r^{2}-\frac{2(2-\nu)}{3(1-\nu)} m(m+1) z^{2}\right]\right. \\
& \left.-Q_{t}^{(4)} r^{-m-1}\left[(m-2) r^{2}+\frac{2(2-\nu)}{3(1-\nu)} m(m-1) z^{2}\right]\right\} \text {, } \\
& 2 G u_{\theta}^{(h, 2)}=z \sum_{t=1}^{\infty} m \sin m \theta\left\{Q_{t}^{(1)} r^{m-1}+Q_{t}^{(2)} r^{-m-1}+Q_{t}^{(3)} r^{m-1}\left[r^{2}-\frac{2(2-\nu)}{3(1-\nu)}(m+1) z^{2}\right]\right. \\
& \left.+Q_{t}^{(4)} r^{-m-1}\left[r^{2}+\frac{2(2-\nu)}{3(1-\nu)}(m-1) z^{2}\right]\right\}, \\
& 2 G u_{z}^{(h, 2)}=\sum_{t=1}^{\infty} \cos m \theta\left\{Q_{t}^{(1)} r^{m}+Q_{t}^{(2)} r^{-m}+Q_{t}^{(3)} r^{m}\left[r^{2}-\frac{2}{1-\nu}(m+1)\left(\frac{h^{2}}{2}-\nu z^{2}\right)\right]\right. \\
& \left.+Q_{t}^{(4)} r^{-m}\left[r^{2}+\frac{2}{1-\nu}(m-1)\left(\frac{h^{2}}{2}-\nu z^{2}\right)\right]\right\}, \\
& \sigma_{r r}^{(h, 2)}=-z \sum_{t=1}^{\infty} \cos m \theta\left\{m(m-1) Q_{t}^{(1)} r^{m-2}+m(m+1) Q_{t}^{(2)} r^{-m-2}+(m+1) Q_{t}^{(3)} r^{m-2}\left[r^{2}\left(m-2+\frac{4}{1-\nu}\right)\right.\right. \\
& \left.\left.-\frac{2(2-\nu)}{3(1-\nu)} m(m-1) z^{2}\right]+(m-1) Q_{t}^{(4)} r^{-m-2}\left[r^{2}\left(m+2-\frac{4}{1-\nu}\right)+\frac{2(2-\nu)}{3(1-\nu)} m(m+1) z^{2}\right]\right\}, \\
& \sigma_{z z}^{(h, 2)}=0 \text {, } \\
& \sigma_{\theta z}^{(h, 2)}=\frac{h^{2}-4 z^{2}}{2(1-\nu)} \sum_{t=1}^{\infty} m \sin m \theta\left[(m+1) Q_{t}^{(3)} r^{m-1}-(m-1) Q_{t}^{(4)} r^{-m-1}\right], \\
& \sigma_{z r}^{(h, 2)}=-\frac{h^{2}-4 z^{2}}{2(1-\nu)} \sum_{t=1}^{\infty} m \cos m \theta\left[(m+1) Q_{t}^{(3)} r^{m-1}+(m-1) Q_{t}^{(4)} r^{-m-1}\right],
\end{aligned}
$$

in which $Q_{t}^{(1)}, \cdots, Q_{t}^{(4)}$ are arbitrary constants to be determined from the boundary conditions on the edges. The required solutions are expressed in the sums of the particular and homogeneous solutions as $u_{r}=u_{r}^{(p)}+u_{r}^{(h, 1)}+u_{r}^{(h, 2)}, \cdots, \sigma_{r \theta}=\sigma_{r \theta}^{(p)}+\sigma_{r \theta}^{(h, 1)}+\sigma_{r \theta}^{(h, 2)}$.

\section{BOUNDARY CONDITIONS}

We consider an annular sector plate whose top and bottom faces are stress-free and whose edges are simply supported. Though the boundary condition of simple support can be prescribed by two different ways, we employ the boundary condition of hinged support here. Then, boundary conditions are as follows :

$$
\begin{aligned}
& \text { on } \quad z= \pm h / 2, \quad \sigma_{z r}=0, \quad \sigma_{z \theta}=0, \quad \sigma_{z z}=0, \\
& (26 \cdot a \sim c) \\
& \text { on } \quad r=a \quad \text { and } \quad r=b, \quad T_{r r}=0, \quad\left(u_{\theta}\right)_{z=0}=0, \quad\left(\frac{\partial u_{\theta}}{\partial z}\right)_{z=0}=0, \quad\left(u_{z}\right)_{z=0}=0, \quad M_{r}=0, \quad \cdots(27 \cdot \mathrm{a} \sim \mathrm{e})
\end{aligned}
$$

in which $T_{r r}$ and $M_{r}$ denote a stress-resultant and a stress-couple, respectively and are expressed as

$$
\begin{aligned}
& T_{r r}=\int_{-h / 2}^{h / 2} \sigma_{r r} d z, \quad M_{r}=\int_{-h / 2}^{h / 2} z \sigma_{r r} d z, \\
& \text { on } \quad \theta= \pm \gamma / 2, \quad T_{\theta \theta}=0, \quad\left(u_{r}\right)_{z=0}=0, \quad\left(\frac{\partial u_{r}}{\partial z}\right)_{z=0}=0, \quad\left(u_{z}\right)_{z=0}=0, \quad M_{\theta}=0,
\end{aligned}
$$

in which $T_{\theta \theta}$ and $M_{\theta}$ denote a stress-resultant and a stress-couple, respectively and are expressed as

$$
T_{\theta \theta}=\int_{-h / 2}^{h / 2} \sigma_{\theta \theta} d z, \quad M_{\theta}=\int_{-h / 2}^{h / 2} z \sigma_{\theta \theta} d z .
$$

We impose the boundary conditions of Eqs. $(26 \cdot a \sim c)$ on Eqs. $(20 \cdot a \sim c)$ from considering that the homogeneous solution in Eqs. $(22 \cdot b)$ and $(24 \cdot b \sim d)$ is not related to the boundary conditions on the top and bottom faces of the plate. Then, the arbitrary constants are determined in the form

$$
B_{t s}^{(1)}=G_{t s}^{(1)}=0 \text {, }
$$




$$
\begin{aligned}
& A_{t s}^{(1)}=-\frac{\alpha E}{4(1-\nu)} \cdot \frac{f_{t s}}{\alpha_{m s}^{2}} \cdot \frac{2(1-\nu)}{\cosh \zeta_{m s}}, \quad L_{t s}^{(1)}=-\frac{\alpha E}{4(1-\nu)} \cdot \frac{f_{t s}}{\alpha_{m s}^{2}} \cdot \frac{2(1-\nu)}{\sinh \zeta_{m s}}, \\
& C_{t s}^{(1)}=-\frac{\alpha E}{4(1-\nu)} \cdot \frac{f_{t s}}{\alpha_{m s}^{2}} \cdot \frac{1}{\cosh \zeta_{m s}}, \quad M_{t s}^{(1)}=-\frac{\alpha E}{4(1-\nu)} \cdot \frac{f_{t s}}{\alpha_{m s}^{2}} \cdot \frac{1}{\sinh \zeta_{m s}} .
\end{aligned}
$$

Thus, the six arbitrary constants except $E_{t}^{(1)}$ and $E_{t}^{(2)}$ contained in the particular solution were exactly determined. By substituting the values of Eqs. $(31 \cdot a \sim e)$ into the particular solution, the components of displacement and the component of stress $\sigma_{r r}^{(p)}$ are expressed as

$$
\begin{aligned}
& 2 G u_{r}^{(\rho)}=-\frac{\alpha E}{2} \sum_{t=1}^{\infty} \sum_{s=1}^{\infty} \frac{f_{t s}}{\alpha_{m s}} \cos m \theta\left[\frac{m}{\alpha_{m s} r} C_{m}\left(\alpha_{m s} r\right)-C_{m+1}\left(\alpha_{m s} r\right)\right]\left(\frac{\cosh \alpha_{m s} z}{\cosh \zeta_{m s}}+\frac{\sinh \alpha_{m s} z}{\sinh \zeta_{m s}}\right) \\
& +2 \beta \sin \beta z \sum_{t=1}^{\infty} m \cos m \theta \frac{1}{\beta r}\left[E_{t}^{(1)} I_{m}(\beta r)+E_{t}^{(2)} K_{m}(\beta r)\right] \text {, } \\
& 2 G u_{\theta}^{(p)}=\frac{\alpha E}{2} \sum_{t=1}^{\infty} \sum_{s=1}^{\infty} \frac{f_{t s}}{\alpha_{m s}} \sin m \theta \frac{m}{\alpha_{m s} r} C_{m}\left(\alpha_{m s} r\right)\left(\frac{\cosh \alpha_{m s} z}{\cosh \zeta_{m s}}+\frac{\sinh \alpha_{m s} z}{\sinh \zeta_{m s}}\right) \\
& -2 \beta \sin \beta z \sum_{t=1}^{\infty} \sin m \theta\left\{E_{t}^{(1)}\left[\frac{m}{\beta r} I_{m}(\beta r)+I_{m+1}(\beta r)\right]\right. \\
& \left.+E_{t}^{(2)}\left[\frac{m}{\beta r} K_{m}(\beta r)-K_{m+1}(\beta r)\right]\right\} \text {, } \\
& 2 G u_{z}^{(p)}=\frac{\alpha E}{2} \sum_{t=1}^{\infty} \sum_{s=1}^{\infty} \frac{f_{t s}}{\alpha_{m s}} \cos m \theta C_{m}\left(\alpha_{m s} r\right)\left(\frac{\sinh \alpha_{m s} z}{\cosh \zeta_{m s}}+\frac{\cosh \alpha_{m s} z}{\sinh \zeta_{m s}}\right) \text {, } \\
& \sigma_{r r}^{(p)}=-\frac{\alpha E}{2} \sum_{t=1}^{\infty} \sum_{s=1}^{\infty} f_{t s} \cos m \theta \frac{1}{\alpha_{m s} r}\left[\frac{m(m-1)}{\alpha_{m s} r} C_{m}\left(\alpha_{m s} r\right)+C_{m+1}\left(\alpha_{m s} r\right)\right]\left(\frac{\cosh \alpha_{m s} z}{\cosh \zeta_{m s}}+\frac{\sinh \alpha_{m s} z}{\sinh \zeta_{m s}}\right) \\
& +2 \beta^{2} \sin \beta z \sum_{t=1}^{\infty} m \cos m \theta \frac{1}{\beta r}\left\{E_{t}^{(1)}\left[\frac{m-1}{\beta r} I_{m}(\beta r)+I_{m+1}(\beta r)\right]\right. \\
& \left.+E_{t}^{(2)}\left[\frac{m-1}{\beta r} K_{m}(\beta r)-K_{m+1}(\beta r)\right]\right\} \text {. }
\end{aligned}
$$

The boundary conditions of Eqs. $(29 \cdot a \sim$ e) are self-evidently satisfied by the cosine function with the argument $m$. If we substitute Eqs. $(22 \cdot a)$ and (33) into Eq. $(28 \cdot a)$, we obtain the stress-resultant $T_{r r}$. By making use of $T_{r r}$, Eqs. $(21 \cdot b)$ and $(32 \cdot b)$, the two boundary conditions of Eqs. $(27 \cdot a$, b) yield a system of linear algebraic equations with four unknowns, i. e., $P_{t}^{(1)}, \cdots, P_{t}^{(4)}$. If we substitute Eqs. (24•a) and (33) into Eq. $(28 \cdot \mathrm{b})$, we obtain the stress-couple $M_{r}$. By making use of $M_{r}$, Eqs. $(23 \cdot \mathrm{b}, \mathrm{c}$ ) and $(32 \cdot b, c)$, the three boundary conditions of Eqs. $(27 \cdot c \sim e)$ yield a system of linear algebraic equations with six unknowns, i. e., $Q_{t}^{(1)}, \cdots, E_{t}^{(2)}$. The arbitrary constants $P_{t}^{(1)}, \cdots, P_{t}^{(4)}$ and $Q_{t}^{(1)}, \cdots, E_{t}^{(2)}$ are determined from their systems of linear algebraic equations which are omitted on account of limited space.

In order to express the components of displacement and stress and the systems of linear algebraic equations in the nondimensional form, it is convenient to replace the arbitrary constants and the Fourier coefficient used in the above with the following ones:

$$
\begin{aligned}
& \frac{P_{t}^{(1)} a^{m-2}}{\alpha E T_{0}}=\bar{P}_{t}^{(1)}, \quad \frac{P_{t}^{(2)} a^{-m-2}}{\alpha E T_{0}}=\bar{P}_{t}^{(2)}, \quad \frac{P_{t}^{(3)} a^{m}}{\alpha E T_{0}}=\bar{P}_{t}^{(3)}, \quad \frac{P_{t}^{(4)} a^{-m}}{\alpha E T_{0}}=\bar{P}_{t}^{(4)}, \quad \frac{Q_{t}^{(1)} a^{m-1}}{\alpha E T_{0}}=\bar{Q}_{t}^{(1)} ; \\
& \frac{Q_{t}^{(2)} a^{-m-1}}{\alpha E T_{0}}=\bar{Q}_{t}^{(2)}, \quad \frac{Q_{t}^{(3)} a^{m+1}}{\alpha E T_{0}}=\bar{Q}_{t}^{(3)}, \quad \frac{Q_{t}^{(4)} a^{-m+1}}{\alpha E T_{0}}=\bar{Q}_{t}^{(4)}, \quad \frac{E_{t}^{(1)} I_{m}(\beta b)}{a^{2} \alpha E T_{0}}=\bar{E}_{t}^{(1)} ; \\
& \frac{E_{t}^{(2)} K_{m+1}(\beta a)}{a^{2} \alpha E T_{0}}=\bar{E}_{t}^{(2)}, \quad \frac{f_{t s}}{T_{0}}=\bar{f}_{t s},
\end{aligned}
$$

in which $T_{0}$ is the constant temperature.

\section{NUMERICAL RESULTS AND DISCUSSION}

Before carrying out numerical calculations, the prescribed surface temperature $g(r, \theta)$ is to be concretely given. As an example, we consider the following temperature which is partially constant in the 
$r$-direction and which follows a quadratic parabola in the $\theta$-direction :

$$
g(r, \theta)= \begin{cases}T_{0}\left(\frac{2}{\gamma}\right)^{2}\left[\left(\frac{\gamma}{2}\right)^{2}-\theta^{2}\right] \text { for } \frac{a+b-c}{2}<r<\frac{a+b+c}{2} \text { and }-\frac{\gamma}{2} \leq \theta \leq \frac{\gamma}{2} \\ 0 \text { for } a \leq r<\frac{a+b-c}{2} \text { or } \frac{a+b+c}{2}<r \leq b \text { and }-\frac{\gamma}{2} \leq \theta \leq \frac{\gamma}{2} .\end{cases}
$$

By substituting the above function into Eq. (11), the Fourier coefficient $f_{t s}$ is expressed as

$$
\begin{aligned}
& f_{t s}=\frac{16 T_{0}}{\pi^{3}} \cdot \frac{(-1)^{(t-1) / 2}}{t^{3}} \cdot \frac{1}{\lambda_{m s}\left[\varkappa^{2} C_{m+1}^{2}\left(\varkappa \lambda_{m s}\right)-C_{m+1}^{2}\left(\lambda_{m s}\right)\right]}\left\{2\left[\omega_{1} C_{m+1}\left(\frac{\lambda_{m s}}{2} \omega_{1}\right)-\omega_{2} C_{m+1}\left(\frac{\lambda_{m s}}{2} \omega_{2}\right)\right]\right. \\
& +m\left[\omega_{1}\left\{C_{m+1}\left(\frac{\lambda_{m s}}{2} \omega_{1}\right) S_{m}\left(\frac{\lambda_{m s}}{2} \omega_{1}\right)-C_{m}\left(\frac{\lambda_{m s}}{2} \omega_{1}\right) S_{m+1}\left(\frac{\lambda_{m s}}{2} \omega_{1}\right)\right\}\right. \\
& \left.\left.-\omega_{2}\left\{C_{m+1}\left(\frac{\lambda_{m s}}{2} \omega_{2}\right) S_{m}\left(\frac{\lambda_{m s}}{2} \omega_{2}\right)-C_{m}\left(\frac{\lambda_{m s}}{2} \omega_{2}\right) S_{m+1}\left(\frac{\lambda_{m s}}{2} \omega_{2}\right)\right\}\right]\right\},
\end{aligned}
$$

in which

$$
\omega_{1}=1+\frac{b}{a}+\frac{c}{a}, \quad \omega_{2}=1+\frac{b}{a}-\frac{c}{a},
$$

and $S_{m}(x)$ and $S_{m+1}(x)$ are Schläfli polynomials expressed as

$$
S_{p}(x)=\pi \sum_{k=-p}^{p}\left[J_{p}(x) Y_{k}(x)-J_{k}(x) Y_{p}(x)\right]
$$

Numerical calculations were carried out for an annular sector plate with the central angle $\gamma=\pi / 2$, outer radius to inner radius, of the circular edge, ratio $b / a=6.0, c / a=2.0$ and Poisson's ratio $\nu=0.25$. In this case, thickness to length of the straight edge ratio, i. e., the thickness-width ratio $e=h / d$ is taken

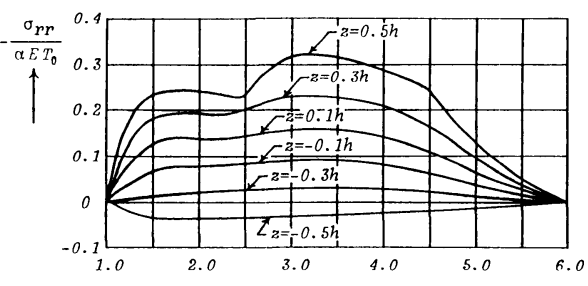

Fig. 2 Stress distribution of $\sigma_{r r}(\theta=0, e=h / d=1 / 4)$.

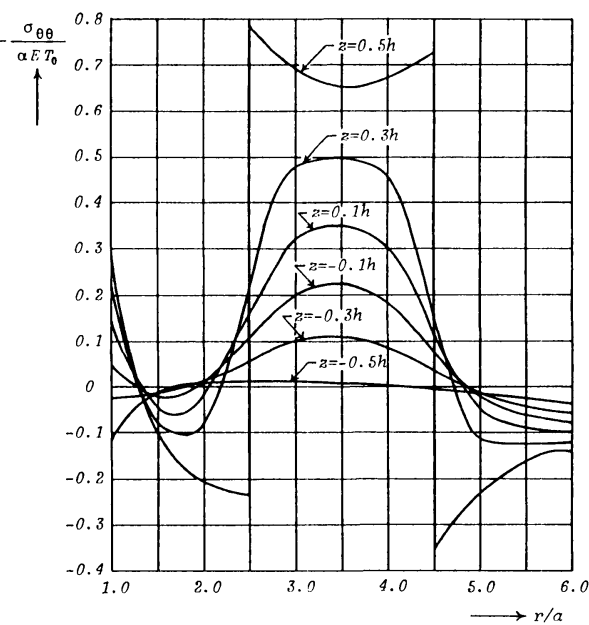

Fig. 3 Stress distribution of $\sigma_{\theta \theta}(\theta=0, \quad e=h / d=1 / 4)$.

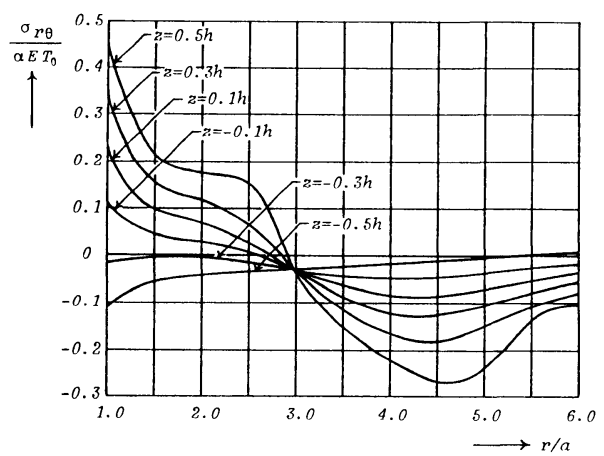

Fig. 4 Stress distribution of $\sigma_{r \theta}(\theta=\pi / 8, \quad e=h / d=1 / 4)$.

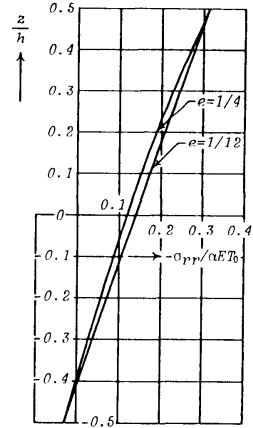

Fig. 5 Stress distribution of $\sigma_{r r}(\theta=0, \quad r / a=3.5$, $e=h / d)$

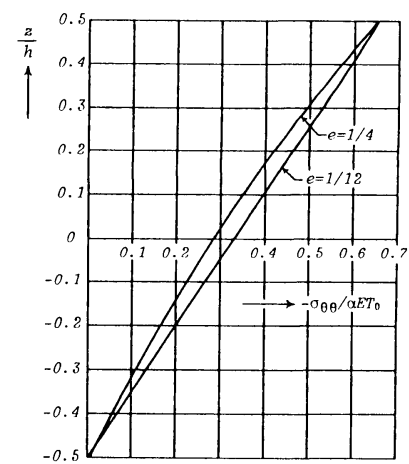

Fig. 6 Stress distribution of $\sigma_{\theta \theta}$ $(\theta=0, \quad r / a=3.5, \quad e=h / d)$. 


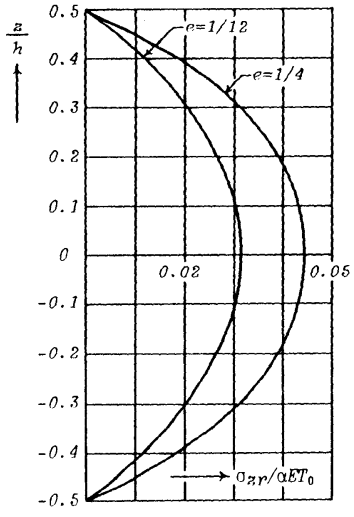

Fig. 7 Stress distribution of $\sigma_{z r}$ $(\theta=0, \quad r / a=1.0, \quad e=h / d)$.

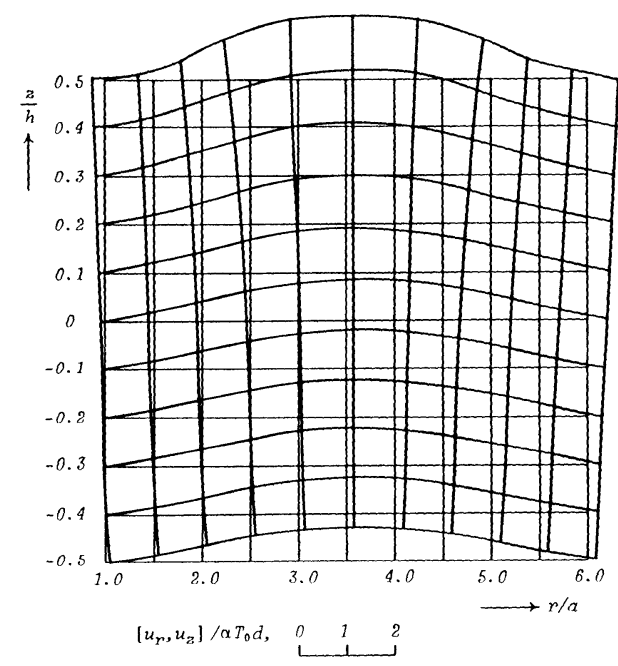

Fig. 8 Shape of deformation $(\theta=0, \quad e=h / d=1 / 4)$.

as $1 / 4$ to $1 / 12$. Numerical results were obtained by taking the first 14 terms for $t$ in an odd number and 68 terms for $s$ in the series. Fig. 2 shows the distribution of normal stress $\sigma_{r r}$ along the $r$-direction on $\theta=0$, in the case of $e=1 / 4$. The value on the top face of the plate, i. e., $z=0.5 \mathrm{~h}$ shows slight discontinuity at the boundaries of the prescribed surface temperature, i. e., $r / a=2.5$ and $r / a=4$.5. The very small values are caused on the inner and outer edges, i. e., $r / a=1.0$ and $r / a=6.0$, because the boundary condition is prescribed by the stress-resultant and the stress-couple instead of the component of stress $\sigma_{r r}$. The value on the bottom face of the plate, i. e., $z=-0.5 h$ is comparatively small in positive. Fig. 3 shows the distribution of normal stress $\sigma_{\theta \theta}$ along the $r$-direction on $\theta=0$, in the case of $e=1 / 4$. The value on $z=0.5 h$ shows remarkable discontinuity at $r / a=2.5$ and $r / a=4$. 5. The value of $\sigma_{\theta \theta}$ is about two times as large as compared to that of $\sigma_{r r}$. Furthermore, all the values on $r / a=6.0$ are positive, and the value on $z=-0.5 h$ is very small in positive or negative. Fig. 4 shows the distribution of shearing stress $\sigma_{r \theta}$ along the $r$-direction on $\theta=\pi / 8$, in the case of $e=1 / 4$. The values near $z=0.5 h$ on $r / a=1.0$ are very large in positive, and the value on $z=-0.5 h$ is comparatively small in negative. Fig. 5 shows the distribution of $\sigma_{r r}$ along the $z$-direction at $\theta=0$ and $r / a=3$. 5. The difference of the values between $e=$ $1 / 4$ and $e=1 / 12$ on the middle plane, i. e., at $z=0$ is $14.8 \%$. Though the difference of the thickness-width ratios is very large, that of the values of $\sigma_{r r}$ is small. The result demonstrates that the influence of the thickness-width ratio on $\sigma_{r r}$ is very little. Fig. 6 shows the distribution of $\sigma_{\theta \theta}$ along the $z$-direction at $\theta=0$ and $r / a=3$. 5. The difference of the values between $e=1 / 4$ and $e=1 / 12$ at $z=0$ is $14.5 \%$, and the influence of the thickness-width ratio on $\sigma_{\theta \theta}$ is similar to $\sigma_{r r}$. Fig. 7 shows the distribution of shearing stress $\sigma_{z r}$ along the $z$-direction at $\theta=0$ and $r / a=1$. 0 . The difference of the values between $e$ $=1 / 4$ and $e=1 / 12$ at $z=0$ is $29.7 \%$. The result demonstrates that the influence of the thickness-width ratio on $\sigma_{z r}$ is more than those on $\sigma_{r r}$ and $\sigma_{\theta \theta}$. Fig. 8 shows the shape of deformation on $\theta=0$, in the case of $e=1 / 4$. The value of displacement $u_{z}$ becomes the largest one at $z / h=0.5$ and $r / a=3.5$. The value of displacement $u_{r}$ becomes the largest one at $z / h=0.5$ and $r / a=6.0$. The warp of the cross section becomes the smallest value near $r / a=3.5$ and grows as $r / a$ approaches 6.0 .

\section{CONCLUSIONS}

An analysis for thermal-bending stresses in an annular sector plate prescribed to surface temperatures on the top and bottom faces and on the simply supported edges was carried out by the theory of moderately thick plates. From the results of the numerical calculations for the plate, the following conclusions may be 
drawn :

(1) The value of normal stress $\sigma_{r r}$ shows slight discontinuity at the boundaries of the prescribed surface temperature on the top face.

(2) The value of normal stress $\sigma_{\theta \theta}$ shows remarkable discontinuity at the boundaries of the prescribed surface temperature on the top face and is about two times as large as compared to that of $\sigma_{r r}$.

(3) The value of shearing stress $\sigma_{r \theta}$ becomes very large in positive near the top of the inner edge and becomes comparatively small in negative on the bottom face.

(4) The influence of the thickness-width ratio on $\sigma_{r r}$ and $\sigma_{\theta \theta}$ are very little.

(5) The influence of the thickness-width ratio on shearing stress $\sigma_{z r}$ is more than those on $\sigma_{r r}$ and $\sigma_{\theta \theta}$.

(6) The value of displacement $u_{z}$ becomes the largest one on the top face, and the value of displacement $u_{r}$ becomes the largest one on the outer edge.

\section{REFERENCES}

1) Woinowsky-Krieger, S. : Der Spannungszustand in dicken elastischen Platten, Ing. -Arch., Band 4, pp. 203-226, 1933.

2) Love, A. E. H. : A Treatise on the Mathematical Theory of Elasticity, p. 455, 4th ed., Dover Pub., 1944.

3) Reissner, E. : The effect of transverse shear deformation on the bending of elastic plates, J. Appl. Mech., Vol. 12, pp. A 69-A 77, 1945.

4) Mindlin, R. D. : Influence of rotatory inertia and shear on flexural motions of isotropic, elastic plates, J. Appl. Mech., Vol. 18, pp. 31-38, 1951.

5) Lo, K. H., Christensen, R. M. and Wu, E.M. : A high-order theory of plate deformation, J. Appl. Mech., Vol.44, pp. 663-668, 1977.

6) Nowinski, J. L. : Theory of Thermoelasticity with Applications, p.407, Sijthoff \& Noordhoff Int. Pub., 1978.

7) Tauchert, T.R. and Aköz, A. Y. : Thermal stresses in an orthotropic elastic slab due to prescribed surface temperatures, J. Appl. Mech., Vol.41, pp. 222-228, 1974.

8) Ishikawa, K., Natsume, S. and Tanimoto, B. : Stationary thermal stresses in orthotropic elastic slabs, Proc. of the JSCE, No. 314, pp. 1-13, 1981 (in Japanese).

9) Noda, N., Takeuti, Y. and Takakura, K. : Transient thermal stress problem in a thick plate due to sectional heat supply on its flat surfaces, Trans. of the JSME, Vol.48, No.427, pp.329-338, 1982 (in Japanese).

10) Sugano, Y. and Kimoto, J. : An analytical solution for asymmetric thermal stresses in a hollow circular plate of variable thickness, Trans. of the JSME, Vol.53, No. 494, pp. 1927-1932, 1987 (in Japanese).

11) Noda, N. and Takeuti, Y. : Transient thermal stresses in a transversely isotropic thick circular plate, Theoretical and Applied Mechanics, Vol. 31, pp. 123-133, 1983.

12) Sugano, Y. : Transient thermal stresses in an anisotropic thick plate with a cylindrical hole, Trans. of the JSME, Vol. 47 , No.419, pp.730-739, 1981 (in Japanese).

13) Okumura, I., Honda, Y. and Yoshimura, J. : On an analysis for circular ring sector plates by one theory of moderately thick plates, Proc. of the JSCE, No. 326, pp. 15-28, 1982 (in Japanese).

14) Okumura, I. A. and Onaka, T. : An expression for solutions to three-dimensional elasticity problems in cylindrical and spherical coordinates, Proc. of the JSCE, No.374/I -6, Structural Eng. /Earthquake Eng., Vol. 3, No. 2, pp. 185-194, 1986.

15) Okumura, I. A. : On some solutions to plane stress problems in cylindrical coordinates, Mem. of the Kitami Institute of Technology, Vol. 12, No. 2, pp. 225-238, 1981 (in Japanese).

(Received April 6 1989) 